\title{
Theorems connecting different classes of Self-Reciprocal Functions
}

\author{
By Brij Mohan Mehrotra.
}

(Received 4th October, 1933; and in revised form 26th February, 1934. Read 3rd March, 1934.)

1. The question, "How, from a given function which is selfreciprocal for a transform of a particular order, can we construct other functions which are self-reciprocal for transforms of different orders?" was first raised by Hardy and Titchmarsh ${ }^{1}$ who gave some rules for constructing such functions. Following their method, I have shown, in a recent paper, ${ }^{2}$ that there are certain general theorems of the following type:-

If $f(x)$ is its own $J_{\mu}$ transform, $g(x)$ is its own $J_{\nu}$ transform. In this note I add a few more such theorems, the interest lying mainly in the results themselves and not in a rigorous proof thereof; and hence only the formal procedure is given here.

For his constant guidance in my work $\mathrm{I}$ wish to express my thanks to Prof. E. C. Titchmarsh at whose suggestion I started the investigation.

2. Following Hardy and Titchmarsh I will say that a function is $R_{\nu}$ if it is self-reciprocal for $J_{\nu}$ transforms, and it is $-R_{\nu}$ if it is skewreciprocal for $J_{\nu}$ transforms. Also, for $R_{l}$ and $R_{-\frac{1}{t}} I$ will write $R_{s}$ and $R_{c}$ respectively.

I will make use of the following result of Hardy and Titchmarsh. ${ }^{3}$

A necessary and sufficient condition that a function $f(x)$ should be $R_{\mu}$ is that it should be of the form

$$
f(x)=\frac{1}{2 \pi i} \int_{c-i \infty}^{c+i \infty} 2^{\frac{1}{3}} \Gamma\left(\frac{1}{4}+\frac{1}{2} \mu+\frac{1}{2} s\right) \psi(s) x^{-8} d s,
$$

where $0<c<1$, and

$$
\psi(s)=\psi(1-s) .
$$

\footnotetext{
1 Hardy and Titchmarsh IV.

2 Mehrotra V.

${ }^{3}$ Hardy and Titchmarsh III, Theorem 8.
} 
3. Theorem I. If $f(x)$ is $R_{1}$, the function

$$
g(x)=x^{-\frac{1}{2} \nu} \int_{0}^{\infty} y^{-\frac{1}{2} \nu} H_{\frac{1}{t}(v-1)}(x y) f(y) d y,
$$

where $H_{\nu}(x)$ is Struve's function of $\operatorname{order}^{1} \nu$, is $R_{\nu}$.

This theorem can be proved on the lines of a similar theorem of Hardy and Titchmarsh', or it can be derived from a more general theorem proved in my paper referred to above ${ }^{3}$, with the help of a formula given by Watson'.

4. Theorem II. If $f(x)$ is $R_{\mu}$, the function

is $R_{\nu}$, provided that

$$
g(x)=\frac{1}{x} \int_{0}^{x} Q\left(\log \frac{x}{y}\right) f(y) d y
$$

$$
\left.\begin{array}{c}
Q(x)=\frac{1}{2 \pi i} \int_{k-i \infty}^{k+i \infty} \Gamma\left(\frac{1}{4}+\frac{1}{2} \mu+\frac{1}{2} s\right) \Gamma\left(\frac{3}{4}+\frac{1}{2} \nu-\frac{1}{2} s\right) \times(s) e^{x s} d s \quad(x>0) \\
=0 \quad(x<0),
\end{array}\right\}
$$

where $k$ is any positive number, and $\chi(s)$ satisfies (2.2).

By (2.1) we have

$$
g(x)=\frac{1}{2 \pi i x} \int_{0}^{x} Q\left(\log \frac{x}{y}\right) d y \int_{c-i \infty}^{c+i \infty} 2^{1 \varepsilon} \Gamma\left(\frac{1}{4}+\frac{1}{2} \mu+\frac{1}{2} s\right) \psi(s) y^{-\varepsilon} d s,
$$

where $\psi(s)=\psi(1-s)$. Hence

$$
\begin{aligned}
g(x) & =\frac{1}{2 \pi i x} \int_{c-i \infty}^{c+i \infty} 2^{j s} \Gamma\left(\frac{1}{4}+\frac{1}{2} \mu+\frac{1}{2} s\right) \psi(s) d s \int_{0}^{x} Q\left(\log \frac{x}{y}\right) y^{-8} d y \\
& =\frac{1}{2 \pi i} \int_{c-i \infty}^{c+\infty \infty} 2^{\frac{1}{\delta}} \Gamma\left(\frac{1}{4}+\frac{1}{2} \mu+\frac{1}{2} s\right) \psi(s) x^{-s} d s \int_{0}^{\infty} e^{(8-1) u} Q(u) d u .
\end{aligned}
$$

Now, using a form of Mellin's Inversion Formula ${ }^{5}$, from (4.1) we have

$$
\int_{0}^{\infty} e^{-x \delta} Q(x) d x=\Gamma\left(\frac{1}{4}+\frac{1}{2} \mu+\frac{1}{2} s\right) \Gamma\left(\frac{3}{4}+\frac{1}{2} \nu-\frac{1}{2} s\right) \chi(s),
$$

where $\chi(s)=\chi(1-s)$. Changing $s$ into $1-s$, we get

$$
\int_{0}^{\infty} e^{(8-1) x} Q(x) d x=\Gamma\left(\frac{3}{4}+\frac{1}{2} \mu-\frac{1}{2} s\right) \Gamma\left(\frac{1}{4}+\frac{1}{2} \nu+\frac{1}{2} s\right) \chi(s) .
$$

\footnotetext{
1 Watson VII, \$10.4(2).

2 Hardy and Titchmarsh IV, § 2.

${ }^{3}$ Mehrotra $V, \S 8$.

4 Watson VII, \$13.24(2).

5 See Bateman I, Hardy II and Pincherle VI.
} 
Hence

$$
\begin{aligned}
g(x) & =\frac{1}{2 \pi i} \int_{c-i \infty}^{c+i \infty} 2^{1 s} \Gamma\left(\frac{1}{4}+\frac{1}{2} \mu+\frac{1}{2} s\right) \Gamma\left(\frac{3}{4}+\frac{1}{2} \mu-\frac{1}{2} s\right) \Gamma\left(\frac{1}{4}+\frac{1}{2} \nu+\frac{1}{2} s\right) \chi(s) \psi(s) x^{-8} d s \\
& =\frac{1}{2 \pi i} \int_{c-i \infty}^{c+i \infty} 2^{\frac{1}{2}} \Gamma\left(\frac{1}{4}+\frac{1}{2} \nu+\frac{1}{2} s\right) \psi_{1}(s) x^{-8} d s,
\end{aligned}
$$

where $\psi_{1}(s)=\Gamma\left(\frac{1}{4}+\frac{1}{2} \mu+\frac{1}{2} s\right) \Gamma\left(\frac{3}{4}+\frac{1}{2} \mu-\frac{1}{2} s\right) \chi(s) \psi(s)$.

As $\psi_{1}(s)$ satisfies the equation

$$
\psi_{1}(s)=\psi_{1}(1-s)
$$

it follows from (2.1) that $g(x)$ is $R_{v}$.

5. If, in Theorem II, we put $\mu=\nu$, we get a corollary. If $f(x)$ is $R_{\nu}$, the function

$$
g(x)=\frac{1}{x} \int_{0}^{x} Q\left(\log \frac{x}{y}\right) f(y) d y
$$

is $R_{v}$ provided that

$$
\begin{array}{rlrl}
Q(x) & =\frac{1}{2 \pi i} \int_{k-i \infty}^{k+i \infty} e^{x s} \lambda(s) d s \quad & (x>0) \\
& =0 \quad(x<0),
\end{array}
$$

where $\lambda(s)=\lambda(1-s)$

6. Theorem III. If $f(x)$ is $R_{\mu}$, the function

is $R_{v}$, provided that

$$
g(x)=\int_{0}^{1 / x} Q\left(\log \frac{1}{x y}\right) f(y) d y
$$

$$
\left.\begin{array}{rl}
Q(x) & =\frac{1}{2 \pi i} \int_{k-i \infty}^{k+i \infty} \Gamma\left(\frac{1}{4}+\frac{1}{2} \mu+\frac{1}{2} s\right) \Gamma\left(\frac{1}{4}+\frac{1}{2} \nu+\frac{1}{2} s\right) \chi(s) e^{x s} d s \quad(x>0) \\
& =0 \quad(x<0),
\end{array}\right\}
$$

where $\chi(s)$ satisfies (2.2).

The proof of this theorem is similiar to that of Theorem II.

The symmetry of the integral in (6.1) shows that if $f(x)$ is $R_{\nu}$, $g(x)$ is $R_{\mu}$.

7. For the particular case $\mu=\mp \frac{1}{2}, \nu= \pm \frac{1}{2}$, the above theorem takes the simpler form:

If $f(x)$ is $R_{c}$ (or $R_{8}$ ), the function

$$
g(x)=\int_{0}^{1 / x} Q\left(\log \frac{1}{x y}\right) f(y) d y
$$


is $R_{8}$ (or $R_{c}$ ) provided that

$$
\begin{aligned}
Q(x) & =\frac{1}{2 \pi i} \int_{k-i \infty}^{k+i \infty} \Gamma(s) \chi(s) e^{x \varepsilon} d s \quad(x>0) \\
& =0 \quad(x<0),
\end{aligned}
$$

where $\chi(s)$ satisfies $(2.2)$.

\section{REFERENCES.}

1. H. Bateman. "Report on the history and present state of the theory of Integral Equations." Reports of the British Assoc. (Sheffield, 1910), 61.

2. G. H. Hardy. "Further notes on Mellin's Inversion Formula." Messenger of Math., 50 (1921), 165-171.

3. G. H. Hardy and E. C. Titchmarsh. "Self-Reciprocal Functions." Quart. Journal of Math., Oxford Series 1 (1930), 196-231.

4. G. H. Hardy and E. C. Titchmarsh. "Formulae connecting different classes of SelfReciprocal Functions." Proc. London Math. Soc., II (33) (1931), 220̃-232.

5. B. M. Mehrotra. "Some Theorems on Self-Reciprocal Functions." Proc. London Math. Soc., II (34) (1932), 231-240.

6. S. Pincherle. "Studi sopra alcune operazioni funzionali." Mem. di Bologma (4), 7 (1886), 393-442.

7. G. N. Watson. Theory of Bessel Functions. Cambridge 1922. 